\title{
Comparative Study between Laparoscopic Tubal Disconnection and Ultrasound Guided Tubal Aspiration in the Management of Hydrosalpinx Prior to ICSI
}

Mohamed Ahmed Hamza Department of Obstetrics and Gynecology,

Faculty of Medicine, Cairo University, Cairo
Corresponding author :

Mohamed Ahmed Hamza Address: 6th of October,Cairo, Egypt

Telephone no.: 01007100166

Email: Dr.hamza.mh@gmail.com

\begin{abstract}
$\underline{\text { Abstract }}$
Objective: To compare the efficacy of ultrasound-guided aspiration with laparoscopic proximal tubal occlusion in the management of hydrosalpinx prior to ICSI.
\end{abstract}

Patients and Methods: This clinical trial included 40 candidates for ICSI suffering from hydrosalpinx. Patients were randomly divided into one of two groups for hydrosalpinx management; $\operatorname{GroupLD}(\mathrm{n}=20)$ bylaparoscopicproximaltubal disconnection and GroupAS( $\mathrm{n}=20)$, byvaginalultrasoundguidedaspiration onthesettingoftheoocyteretrievalfortheICSIcycle. The primary and secondary outcome measures were clinical and chemical pregnancy rates, respectively.

Results: The two groups were comparable regarding basic clinical characteristics and results of ovarian stimulation and oocyte retrieval. The proportions of clinical pregnancies and chemical pregnancies were comparable in the two groups $(\mathrm{p}=0.342$ and 0.525 , respectively). Eleven pregnancies were diagnosed chemically in laparoscopic disconnection group compared to 8 in aspiration group. In laparoscopic disconnection group one pregnancy failed to continue clinically.

Conclusion: Aspiration of hydrosalpingeal fluid at the time of oocyte retrieval is as effective as laparoscopic disconnection of the fallopian tube in terms of clinical pregnancy rate after ICSI cycles.

\section{$\underline{\text { Introduction }}$}

Tubal pathology accounts for $14 \%$ of the causes of subfertility. The most severe manifestation of tubal disease is hydrosalpinx[1]. An increased risk for early pregnancy loss and increased risk for ectopic pregnancies were reported $[2,3,4]$.

A paradox emerged after recognition that IVF in patients with tubal disease was associated with lower implantation rates and an increased risk of early pregnancy loss than after IVF in other subfertile patients [5][6]. This deleterious effect of tubal disease on IVF outcome may be related to the severity of tubal damage [7].

The mechanism of this deleterious effect on IVF outcome is not clear. The hydrosalpinx fluid seems to have a key role [8]. It may affect the transferred embryo; possibly by embryotoxic factors [9]. The bathing of the endometrial cavity in hydrosalpinx fluid may interfere with the endometrial interaction with the transferred embryo necessary for implantation [10,11]. The presence of a thin layer of fluid upon the endometrial surface [12] and changes in endometrial peristalsis by the fluid [13] may wash-out or hinder implantation of the transferred embryo.

Many management options were suggested as proximal tubal occlusion. Other options include hysteroscopic tubal occlusion, ultrasound guided aspiration of hydrosalpinx and medical treatment (antibiotics and/ 
or corticosteroids). Several authors suggest that ultrasound guided aspiration of hydrosalpinx fluid is the best alternative because it is simple, safe, easy and inexpensive. Furthermore, there is evidence supporting its beneficial effect on the outcomes of IVF-ET from several prospective randomized controlled studies $[14,15]$.

The aim of the current study was to compare the efficacy of ultrasound-guided aspiration with laparoscopic proximal tubal occlusion in the management of hydrosalpinx prior to ICSI to assess the impact of treatment on ICSI cycle outcome.

\section{Patients and Methods:}

This clinical trial was conducted at the Obstetrics and Gynecology Department, Kasr El Aini Hospital, Cairo University. The study involved 40 patients with tubal factor of infertility having hydrosalpinx and candidates for ICSI cycles.

Inclusion criteria were age 20-30 years, primary or secondary infertility, tubal factor of infertility with uni- or bilateral hydrosalpinx and scheduled for ICSIcycle usinglongprotocolforcontrolled ovarian stimulation. Patients with malefactorofinfertility, uterinefactorofinfertility, poor ovarianreserve, poorresponse, obesity (BMI > $30 \mathrm{~kg} / \mathrm{m} 2$ ) or any medical disorder were excluded from the study.

All patients were subjected to careful history raking, general and local examination. A recent hysterosalpingography (HSG) done within the last 6 months was used to confirm the presence of unilateral or bilateral fallopian tube dilatation with loss of rugal folds without or with decreased contrast in the peritoneal cavity. If HSG was not available, it was done during clinical evaluation

Transvaginal sonography (TVS) was performed using a $7.5 \mathrm{MHz}$ vaginal probe of Sonoace X6 ultrasound machine before laparoscopic management and two weeks after the operation. The uterus was scanned for detection of any endometrial abnormality, visible hydrosalpinx in the form of elongated, diluted, tortuous tube containing fluid which is anechoic was recorded.

The participants were randomized into one of two treatment groups:

- GroupLD(n=20):Inthisgrouphydrosalpinxwas managedbylaparoscopicproximaltubal disconnection prior to the ICSI cycle.

- GroupAS(n=20): Inthisgrouphydrosalpinx was managedbyvaginalultrasoundguidedaspiration onthesettingoftheoocyteretrievalfortheICSIcycle.
Laparoscopic assessment of the peritoneal cavity:

Under General anesthesia, $\mathrm{CO} 2$ pneumoperitoneum and introduction of at least two ports was used to detect:

- Presence or absence of endometriosis.

- Peritoneal spill after cervical cannulation with methylene blue.

- Tubal disconnection using bipolar diathermy or salpingectomy

Technique of ovarian hyperstimulation and embryo transfer:

1. The induction protocol was the long luteal phase agonist protocol. Participants received (GnRHa) long protocol, Decapeptyl $0.1 \mu \mathrm{g}$ SC injection daily starting on day 21 . After pituitary down regulation had been confirmed, by serum E $2<50 \mathrm{pg} / \mathrm{ml}, 225-$ $300 \mathrm{IU}$ of hMG per day was started on day 3 of the cycle, then the dose was adjusted according to the response, being monitored by ultrasound on day 8 or 9 to establish the number of ovarian follicles.

2. Triggering of ovulation was done by 10000 units of hCG IM when two or more follicles reach $18 \mathrm{~mm}$ in mean diameter.

3. Ovum retrieval using transvaginal ultrasound was scheduled 34-36 hours after hCG injection

4. All grade embryos were transferred on day 3-5 after ovum retrieval.

5. Serum B-hCG test was done to confirm pregnancy two weeks after embryo transfer (chemical pregnancy).

6. Transvaginal ultrasound examination was done after 5 weeks from embryo transfer to confirm positive fetal pulsations (clinical pregnancy).

The following parameters were monitored: a) number of oocytes collected in ICSI cycle, b) degree of oocyte maturity, c) endometrial thickness on day of triggering in ICSI cycle and d) number and quality of embryos transferred.

\section{Technique of Laparoscopic tubal disconnection}

Under general anesthesia, using $25 \mathrm{~mm}$ ports for entry at the right and left lower quadrants, the affected fallopian tube(s) was identified. The tube was grasped and the bipolar diathermy was applied $2-3 \mathrm{~cm}$ from the cornu followed by cutting of the diathermized point using scissors

Ultrasound Guided Aspiration was doneunder general anesthesia after ovum pickup during the ICSI cycle. 
Through TVS the aspiration needle is washed from the inside using saline and the same needle was used to aspirate the hydrosalpinx either unilateral or bilateral.

The primary outcome measure was clinical pregnancy rate of the ICSI cycles. The secondary outcome measure was the chemical pregnancy rate.

\section{$\underline{\text { Statistical methods }}$}

Data was analyzed using IBM SPSS Advanced Statistics version 22.0 (SPSS Inc., Chicago, IL). Numerical data were expressed as mean and standard deviation or median and range as appropriate. Qualitative data were expressed as frequency and percentage. Chi-square test was used to examine the relation between qualitative variables. For quantitative data, comparison between the 3 groups was done using student t-test or MannWhitney as appropriate. All tests were two-tailed. A p-value $<0.05$ was considered significant.

\section{$\underline{\text { Results }}$}

The two groups were comparable regarding age, body mass index (BMI), antral follicle count (AFC), and pretreatment hormonal profile as shown in table 1 .

The outcome of ICSI cycles is shown in table 2. During oocyte retrieval, the total number of oocytes ranged from 9 to 16 with no significant difference between the two groups $(\mathrm{p}=0.091)$. These ova were mainly in the mature metaphase I and II with no significant difference between the two groups in the number of MI oocytes $(p=0.195)$ or MII oocytes $(p=0.789)$. There was no significant difference between the two groups in the total number of embryos $(p=0.575)$ or grade A embryos $(p=0.317)$. The proportions of clinical pregnancies and chemical pregnancies were comparable in the two groups $(\mathrm{p}=0.342$ and 0.525 , respectively). Eleven pregnancies were diagnosed chemically in laparoscopic disconnection group compared to 8 in aspiration group. In laparoscopic disconnection group one pregnancy failed to continue clinically.

\section{Discussion}

This study demonstrates that laparoscopic disconnection of the fallopian tube and ultrasound-guided aspiration of hydrosalpinx resulted in comparable pregnancy rates after ICSI cycles.

Salpingectomy remains the most frequently undertaken procedure. However, it can possibly affect ovarian function due to compromising blood supply. Laparoscopic proximal tubal division was suggested to preserve ovarian function. This was the type of laparoscopic salpingectomy adopted in the current study. Laparoscopic proximal tubal division has been previously suggested as an optimal operation method for infertility patients with hydrosalpinges[16]. Other investigators reported similar findings with no effect on ovarian reserve $[17,18,19]$.

Similar to the current study, [20]investigated the effectiveness of proximal tubal cauterization for the treatment of hydrosalpinges before in vitro fertilization. They concluded that proximal tubal cauterization is effective in reversing the adverse effects of hydrosalpinges. Compared to salpingectomy, aspiration, salpingostomy and tubal occlusion are thought to have the advantage of being less invasive, safer and easier to perform in the case of dense adhesions, with shorter hospital stays [19,21].

Aspiration of the hydrosalpinx fluid (HSF) was another choice we used in the current study. It is a rather simple procedure that can overcome some obstacles encountered when planning for open or laparoscopic salpingectomy. In the current study, aspiration of HSF was statistically comparable to laparoscopic salpingectomy. However, it was associated with lower ongoing pregnancy rate $(40 \%)$.

[22] reported that the aspiration of pelvic inflammatory cystic masses resulted in increased ovarian response and a significant increase in the number of embryos per transfer in IVF-ET cycles. [23] reported a patient with bilateral hydrosalpinges who failed to conceive in the first IVF-ET cycle. The patient conceived after ultrasound-guided aspiration of hydrosalpinges which was performed 1 month before the second IVF-ET cycle. [24] reported seven women undergoing 11 IVFET cycles had their hydrosalpinges aspirated at the time of oocyte retrieval without any noted morbidity, resulting in two ongoing pregnancies and three pregnancy losses.

A comparative, controlled retrospective analysis was performed for women with infective tubal damage who were going to have surgical drainage of the hydrosalpinx at the time of oocyte collection for in-vitro fertilization. A total of 237 embryo transfer cycles in women with hydrosalpinges were compared with 705 embryo transfer cycles in women with tubal disease but no hydrosalpinx. Success rates were higher in the first cycle, but did not significantly influence overall differences. The study showed marked reduction in embryo implantation in the presence of tubal damage with distal occlusion, even in the absence of obvious fluid distension. The authors recommended surgical drainage of distended hydrosalpinges in these cases [25]. 
Another study compared clinical pregnancy rate in women with hydrosalpinges with and without aspiration of HSF at the time of oocyte retrieval. It was found that aspiration of hydrosalpinges was associated with a higher clinical pregnancy rate, a higher ongoing pregnancy rate, and a higher implantation rate. This study confirms the association between the presence of hydrosalpinges and poor IVF outcomes [26].

A randomized controlled trial was done on the effects of ultrasound-guided HSF aspiration of ultrasonically diagnosed hydrosalpinx during oocyte collection on IVF outcome including 66 women. Aspiration resulted in a greater biochemical pregnancy rate [15].

More recently, 110 women with ultrasound-visible hydrosalpinges were enrolled in a study to test the effect of ultrasound-guided aspiration of HSF at the time of oocyte retrieval on the outcomes of IVF-ET. The authors reported that patients who underwent aspiration of hydrosalpinges demonstrated a significantly increased implantation and clinical pregnancy rates. In the aspiration group, re-accumulation of HSF within 2 weeks was associated with lower - but not statistically significant - implantation and pregnancy rates, compared to those with no reaccumulation[14].

A recent case report of a 36-year old nullipara with unilateral hydrosalpinx was published. The patient declined salpingectomy prior to IVF treatment and had two failed IVF cycles. Following transvaginal ultrasound scan guided aspiration of the hydrosalpinx fluid at the time of oocyte retrieval, she became pregnant with the third IVF attempt [27].

There are concerns about the possible occurrence of infection after puncture of hydrosalpinx during aspiration of hydrosalpingeal fluid and that rapid reaccumulation of HSF may preclude any beneficial effect of aspiration. In the current study, we did not record any case of flaring of pelvic infection, peritonitis or fluid re-accumulation. These results are in accordance with previous studies reporting no infectious morbidity in patients after aspiration of hydrosalpinges $[15,25,26]$.

A limiting factor in our study is the small number of cases.

In conclusion, the aspiration of hydrosalpingeal fluid at the time of oocyte retrieval is simple, easy, safe and effective procedure for patients with ultrasoundvisible hydrosalpinges. It is as effective as laparoscopic disconnection of the fallopian tube in terms of clinical pregnancy rate after ICSI cylces. Further multicentric prospective randomized studies are needed to confirm these findings.

Table 1: Demographic and clinical characteristics of the two studied group.

\begin{tabular}{lccc}
\hline & $\begin{array}{c}\text { Group LD } \\
\mathbf{n = 2 0}\end{array}$ & $\begin{array}{c}\text { Group AS } \\
\mathbf{n = 2 0}\end{array}$ & p value \\
\hline Age (years) & $25.4 \pm 2.9$ & $24.8 \pm 2.8$ & 0.509 \\
Body mass index (kg/m2) & $22.4 \pm 2.6$ & $21.8 \pm 2.1$ & 0.463 \\
FSH (IU/mL) & $4.7 \pm 1.2$ & $4.3 \pm 1.2$ & 0.327 \\
LH (IU/mL) & $3.8 \pm 1.0$ & $3.4 \pm 0.9$ & 0.242 \\
Prolactin (ng/mL) & $16.3 \pm 4.5$ & $15.9 \pm 4.1$ & 0.807 \\
Estradiol (pg/mL) & $38.4 \pm 15.2$ & $39.2 \pm 12.7$ & 0.849 \\
AMH (ng/mL) & $4.6 \pm 1.5$ & $4.5 \pm 1.6$ & 0.735 \\
Antral follicle count (AFC) & $14(8-17)$ & $12(7-17)$ & 0.091
\end{tabular}

Data presented as mean $\pm \mathrm{SD}$ or median (range)

ase I, MII: metaphase II

Data presented as median (range) 
Table 2: The outcome of ICSI cycle after treatment in the two studied groups.

\begin{tabular}{lccc}
\hline & $\begin{array}{c}\text { Group LD } \\
\mathbf{n = 2 0}\end{array}$ & $\begin{array}{c}\text { Group AS } \\
\mathbf{n = 2 0}\end{array}$ & p value \\
\hline Total No Ova Retrieved & $13(9-16)$ & $13(10-16)$ & 0.091 \\
No. of Atretic ova & $0(0-2)$ & $0(0-2)$ & 0.414 \\
No. of GV oocytes & $0(0-4)$ & $1(0-3)$ & 0.262 \\
No. of MI oocytes & $4(3-7)$ & $5(3-7)$ & 0.195 \\
No. of MII oocytes & $6(5-10)$ & $7(4-11)$ & 0.789 \\
Total No of embryos & $7(4-10)$ & $8(5-11)$ & 0.575 \\
Grade A & $6(3-9)$ & $6(4-8)$ & 0.317 \\
Grade B & $1(0-3)$ & $1(0-3)$ & 0.061 \\
Grade C & $1(0-3)$ & $1(0-3)$ & 0.733 \\
Clinical Pregnancy & $10(50.0 \%)$ & $8(40.0 \%)$ & 0.342 \\
Chemical Pregnancy & $11(55.0 \%)$ & $8(40.0 \%)$ & 0.525 \\
\hline
\end{tabular}

GV: germinal vesicle phase, MI: metaphase 1

\section{References}

1. Evers JL. Female subfertility. Lancet. 2002;360(9327):151-9.

2. Andersen A, Yue Z, Meng F, et al. Low implantation rate after in vitro fertilisation in patients with hydrosalpinges diagnosed by ultrasonography. Hum. Reprod. 1994;9:1935-8.

3. Strandell A. The assessment of endometrial pathology and tubal patency: a comparison between the use of ultrasonography and X-ray hysterosalpingography for the investigation of infertility patients. Ultrasound Obstet Gynecol. 1999; 14:200-4.

4. Vandromme J, Chasse E, Lejeune B, Van Rysselberge M, Delvigne A, Leroy F. Hydrosalpinges in in-vitro fertilization: an unfavourable prognostic feature. Hum Reprod. 1995;10(3):576-9.

5. Camus E, Poncelet C, Goffinet F, Wainer B, Merlet F, Nisand I, et al. Pregnancy rates after in-vitro fertilization in cases of tubal infertility with and without hydrosalpinx: a meta-analysis of published comparative studies. Hum Reprod. 1999; 14:12439.

6. Zeyneloglu HB, Arici A, Olive DL. Adverse effects of hydrosalpinx on pregnancy rates after in vitro fertilization - embryo transfer. FertilSteril. 1998;70:492-9.

7. Csemiczky G, Landgren BM, Fried G,
Wramsby H. High tubal damage grade is associated with low pregnancy rate in women undergoing in vitro fertilisation treatment. Hum Reprod 1996;11:2438-40.

8. Strandell A, Lindhard A. Why does hydrosalpinx reduce fertility; the importance of hydro salpingeal fluid. Hum Reprod. 2002;17(5):1141-5.

9. Mukhurjee T, Copperman AB, McCaffrey C. Hydrosalpinx fluid has embryotoxic effects on murine embryogenesis: a case for prophylactic salpingectomy. FertilSteril1996;66:851-3.

10. Fleming C, Hull MGR. Impaired implantation after in vitro fertilisation treatment associated with hydrosalpinx. $\mathrm{Br} \quad \mathrm{J}$ ObstetGynaecol. 1996;103(3):268-72..

11. Meyer WR, Castelbaum AJ, Somkuti S, Sagoskin AW, Doyle M, Harris JE, Lessey BA. Hydrosalpinges adversely affect markers of endometrial receptivity. Hum Reprod. 1997;12(7):1393-8.

12. Sharara FI. The role of hydrosalpinx in IVF: simply mechanical?. Human Reproduction 1999;14:577-8.

13. Eytan O, Azem F, Gull I, Wolman I, Elad D, Jaffa AJ. The mechanism of hydrosalpinx in embryo implantation. Hum Reprod. 2001;16:2662-7.

14. 14. Fouda UM, Sayed AM. Effect of ultrasoundguided aspiration of hydrosalpingeal fluid during oocyte retrieval on the outcomes of in vitro fertilisation-embryo transfer: a 
randomised controlled trial. Gynecol Endocrinol. 2011;27(8):562-7.

15. 15. Hammadieh N, Coomarasamy A, Ola B, Papaioannou S, Afnan M, Sharif K. Ultrasoundguided hydrosalpinx aspiration during oocyte collection improves pregnancy outcome in IVF: a randomized controlled trial. Hum Reprod. 2008;23(5):1113-7.

16. 16. Nakagawa K, Ohgi S, Nakashima A, Horikawa T, Irahara M, Saito H. Laparoscopic proximal tubal division can preserve ovarian reserve for infertility patients with hydrosalpinges. J ObstetGynaecol Res. 2008;34(6):1037-42.

17. 17. Gelbaya TA, Nardo LG, Fitzgerald CT, Horne G, Brison DR, Lieberman BA. Ovarian response to gonadotropins after laparoscopic salpingectomy or the division of fallopian tubes for hydrosalpinges. FertilSteril. 2006;85(5):1464-8.

18. 18. Sagoskin AW, Lessey BA, Mottla GL, Richter KS, Chetkowski RJ, Chang AS, Levy MJ, Stillman RJ. Salpingectomy or proximal tubal occlusion of unilateral hydrosalpinx increases the potential for spontaneous pregnancy. Hum Reprod. 2003;18(12):2634-7.

19. 19. Surrey ES, Schoolcraft WB. Laparoscopic management of hydrosalpinges before in vitro fertilization-embryo transfer: salpingectomy versus proximal tubal occlusion. FertilSteril. 2001;75(3):612-7.

20. 20. Stadtmauer LA, Riehl RM, Toma SK, Talbert LM. Cauterization of hydrosalpinges before in vitro fertilization is an effective surgical treatment associated with improved pregnancy rates. Am J Obstet Gynecol. 2000;183(2):367-71.

21. 21. Taylor RC, Berkowitz J, McComb PF. Role of laparoscopic salpingostomy in the treatment of hydrosalpinx. FertilSteril. 2001;75(3):594-600.

22. 22. Aboulghar MA, Mansour RT, Serour GI, Sattar MA, Awad MM, Amin Y. Transvaginal ultrasonic needle guided aspiration of pelvic inflammatory cystic masses before ovulation induction for in vitro fertilization. FertilSteril1990;53:311-314.

23. 23. Russell JB, Rodriguez Z, Komins JI. The use of transvaginal ultrasound to aspirate bilateral hydrosalpinges prior to in vitro fertilization: a case report. J In Vitro Fertil Embryo Transfer 1991;8:213-214.

24. 24. Sharara FI, Scott RT, Marut E, Queenan JT Jr. In-vitro fertilization outcome in women with hydrosalpinx. Hum Reprod 1996;11:526-530.

25. 25. Sowter MC, Akande VA, Williams JA, Hull MG. Is the outcome of in-vitro fertilization and embryo transfer treatment improved by spontaneous or surgical drainage of a hydrosalpinx? Hum Reprod1997; 12:2147-2150.

26. 26. Van Voorhis BJ, Sparks AE, Syrop CH, Stovall DW. Ultrasound-guided aspiration of hydrosalpinges is associated with improved pregnancy and implantation rates after in-vitro fertilization cycles. Hum Reprod1998;13:736-739.

27. 27. Okohue JE, Ikimalo JI. IVF Pregnancy and Delivery following Ultrasound Scan Guided Aspiration of a Left Hydrosalpinx- A Case Report. Niger Postgrad Med J. 2015;22(2):123-5. 